\title{
Internações por Diabetes Mellitus como diagnóstico principal na Rede Pública do Brasil, 1999-2001
}

\author{
Hospitalization for Diabetes Mellitus \\ as the first-listed diagnosis in the \\ Brazilian National Health System \\ (SUS), 1999-2001
}

\section{Roger dos Santos Rosa' \\ Maria Inês Schmidt ${ }^{1}$ \\ Bruce Bartholow Duncan' \\ Maria de Fátima Marinho de Souza² \\ André Klafke de Lima' \\ Lenildo de Moura ${ }^{1,2}$ \\ 1 Universidade Federal do Rio Grande do Sul, Programa de Pós-graduação em Epidemiologia e Departamento de Medicina Social \\ ${ }^{2}$ Secretaria de Vigilância em Saúde, Ministério da Saúde}

O trabalho é parte da "Tese de Doutoramento Diabetes Mellitus: magnitude das hospitalizações na rede pública do Brasil, 1991-2001." defendida no Programa de Pós-graduação em Epidemiologia da UFRGS em 2006 por Roger dos Santos Rosa.

Correspondência: Roger dos Santos Rosa. Fernando Abbott, 830 - Porto Alegre, RS CEP 91040360. E-mail: roger.rosa@bcb.gov.br

\section{Resumo}

Objetivo: Descrever no âmbito nacional e por faixa etária, sexo e região as 327.800 hospitalizações por diabetes mellitus (DM) da rede pública do Brasil, entre 1999-2001. Métodos: Foram utilizados dados do Sistema de Informação Hospitalar do Sistema Único de Saúde (SIH/SUS) referentes ao DM (diagnóstico principal CID-10 E10E14 combinado com procedimento realizado) e indicadores anuais de hospitalizações $/ 10^{4}$ hab. e óbitos hospitalares/ $10^{6}$ hab. (ajustados pelo método direto por idade), letalidade, médias de permanência e gastos por internação e por $10^{4} \mathrm{hab}$. em US\$, e regressão logística múltipla para desfecho óbito. Resultados: Houve mais hospitalizações anuais do sexo feminino $\left(7,5 / 10^{4}\right.$ hab. [intervalo de confiança de 95\%: 7,4-7,6] vs. masculino $\left(5,2 / 10^{4}\right.$ hab. [5,2-5,3]), mais óbitos hospitalares anuais de mulheres $\left(38,1 / 10^{6}\right.$ hab. [36,8-39,3] vs. 30,7 [29,5-32,0]), porém maior letalidade no sexo masculino (5,9 vs. $5,0 \%$ ) em todas as regiões. Observou-se incremento das hospitalizações com a idade, mais acentuado para mulheres. Não houve diferença na permanência das internações com óbito $(6,5$ dias $[6,3-6,6])$ ou sem $(6,4[6,3-6,6])$, apesar do gasto por internação superior (US\$ 275,27 [268,37-282,16] vs. 143,45 [136,56-150,35]). O gasto anual/104hab. equivaleu a US\$ 969,09. A razão de chances de óbito hospitalar aumentou com a idade, foi maior para homens $(1,21$ [1,17-1,24]) e 2 vezes maior para habitantes das regiões Nordeste e Sudeste comparados aos da região Sul. Os gastos anuais/10 $\mathrm{hab}$. foram $50-100 \%$ maiores nas regiões mais desenvolvidas. Conclusões: O volume e a desigualdade nos gastos enfatizam a necessidade de cobertura mais adequada da população, evitando as hospitalizações e suas complicações.

Palavras-chave: Diabetes/epidemiologia. Morbidade. Hospitalizações. Recursos em saúde. Acesso aos serviços de saúde. 
Abstract

Aim: To describe at the national level and by age group, gender, and geographic region, all 327,800 first-listed hospitalizations for diabetes mellitus (DM) financed by the national health system (SUS) in Brazil, 1999-2001. Method: Data from the SUS' Hospital Information System (SIH/SUS) for $\mathrm{DM}$ as the first-listed diagnosis (ICD-10 E10-E14 and procedure performed) were obtained and indicators calculated by region of residence of the patients and gender (adjusted by direct method for age with 95\% confidence intervals), age intervals, average length of stay and expenditure by admission and population in US\$. Multiple logistic regression analysis was performed for death as outcome. Results: Hospitalization coefficients were higher for women (7.5/104inhab. [7.4-7.6] vs 5.2 [5.2-5.3]), although hospital lethality was higher for men $(5.9$ vs $5.0 \%)$ in all five regions. No differences were found in the average length of stay between hospitalizations with/without death (6.5 days [6.3-6.3] vs. 6.4 [6.3-6.6]) despite higher average expenditures (US\$ 275.27 [268.37-282.16] vs. 143.45 [136.56150.35]). Population expenditure was US\$ $969.09 / 10^{4}$ inhabitants. Odds ratio for death were higher for men, patients $\geq 75 \mathrm{yrs}$, and inhabitants of the northeast and southeast. Developed regions accounted for nearly twice higher expenses than other regions. Conclusions: Inequalities in hospitalizations and resources emphasize the need for a better health coverage that could reduce the number of hospitalizations and lessen the severity of DM and its complications.

Keywords: Diabetes/epidemiology. Morbidity. Hospitalization. Health resources. Health services accessibility

\section{Introdução}

O diabetes representa um problema importante em diversos países pela carga de sofrimento, incapacidade, perdas de produtividade e morte prematura que provoca ${ }^{1}$. Estima-se que aumentará na população mundial, devido à transição demográfica, de 2,8\% (171 milhões de pessoas) para 4,4\% (366 milhões), entre os anos de 2000 e $2030^{2}$, particularmente de maneira rápida na América Latina ${ }^{3}$. No Brasil, o diabetes mellitus (DM) atingia 7,6\% dos adultos entre 30 e 69 anos nos fins da década de $1980^{4}$. Na Campanha Nacional de Detecção de DM em 2001, 16\% dos examinados ( $\geq 40$ anos) apresentaram glicemia capilar $\geq 100 \mathrm{mg} / \mathrm{dl}$ em jejum ou $\geq 140 \mathrm{mg} / \mathrm{dl}$ em amostra casual ${ }^{5}$. O envelhecimento e o aumento dos níveis de obesidade da população brasileira ${ }^{6}$ observados nos anos 90 certamente têm contribuído para elevar a prevalência do DM.

O manejo adequado do DM no nível de atenção básica reduziria os efeitos econômicos adversos para famílias, comunidades e sociedade em geral, provocado por internações e, principalmente, reduziria as seqüelas e complicações. Neste sentido, o estudo das internações pode auxiliar como indicador da efetividade dos cuidados atuais e das intervenções implementadas ${ }^{7}$. Revisamos a literatura utilizando as palavras-chave "diabetes mellitus" e "hospitalização", sem delimitadores temporais ou de idioma, tanto na base Literatura Latino-Americana em Ciências da Saúde (LILACS) da Biblioteca Virtual de Saúde (BVS) quanto na Biblioteca Nacional de Medicina dos Estados Unidos (PubMed). Não encontramos estudos sobre internações por "diabetes mellitus" no Sistema de Informações Hospitalares do Sistema Único de Saúde (SIH/SUS) com abrangência nacional, particularmente que apresentassem comparações regionais.

O impacto do DM no sistema de saúde, a diversidade de estrutura e de funcionamento das redes de atenção no território brasileiro e a transferência de respon- 
sabilidades do manejo dessa condição para o nível local no sistema público de saúde motivaram este trabalho. Objetivase estudar a magnitude das hospitalizações por DM na rede pública de saúde brasileira em anos recentes. Tenciona-se analisar volume, mortalidade e letalidade hospitalares, média de permanência e gasto médio por internação e populacional, segundo as regiões brasileiras, o sexo e as faixas etárias selecionadas. Nesta etapa, serão apresentados somente os resultados considerando o DM como diagnóstico principal.

\section{Métodos}

A fonte dos dados estudados foram os arquivos do tipo reduzido ("RD") do Sistema de Informações Hospitalares do Sistema Único de Saúde (SIH/SUS) ${ }^{8}$, que controla os pagamentos dos serviços prestados por hospitais públicos e privados ao sistema público de saúde brasileiro. Os dados originaram-se do formulário padronizado Autorização de Internação Hospitalar (AIH), usado pelos gestores públicos desde a década de 80 para pagamento prospectivo com base em grupos de diagnósticos relacionados (DRG - "Diagnostic Related Groups”) com algumas adaptações ${ }^{9}$. As hospitalizações são em número um pouco superior ao de indivíduos internados, pois uma mesma pessoa pode ser internada mais de uma vez pelo mesmo motivo no período aqui considerado.

A partir de 37 milhões de internações financiadas pelo Sistema Único de Saúde (SUS) do Brasil no período 1999-2001, identificaram-se preliminarmente todas as 372.358 internações (1\%) com diagnóstico principal (motivo da hospitalização) de $d i$ abetes mellitus (categorias E10 a E14 da Classificação Internacional de Doenças $10^{a}$ revisão (CID-10)). Para aumentar a precisão diagnóstica e permitir comparações internacionais, restringiu-se a 327.800 internações $(0,9 \%)$, todas do Tipo 1 (AIH-1 normal, ou seja, não abrangem prorrogações), cujos procedimentos realizados fo- ram 322.171 hospitalizações para "tratamento de diabetes sacarino" (códigos 82.300-04-6 e 82.500-05-3 do SIH/SUS) ou 5.629 internações para "diagnóstico e/ou primeiro atendimento clínico (72-500-000 ) ou pediátrico (71.300-00-7)”. As 44.558 exclusões $(0,1 \%)$ referiam-se a 9.580 de tratamento de diabetes insípido, 20.735 cirurgias de amputação de segmentos dos membros inferiores, 4.825 procedimentos cirúrgicos da pele e/ou subcutâneo e 9.418 internações relativas a outros 560 procedimentos cuja maior freqüência individual foi de 1,3\%. Não foram incluídas internações por DM na gestação (categoria O24 na CID-10).

Para estabilizar flutuações anuais, apurou-se o volume médio de internações e de óbitos hospitalares no triênio por região de residência do paciente, sexo e idade no momento da hospitalização. A idade foi categorizada em 5 faixas etárias selecionadas (0-19 anos, 20-44, 45-64, 65-74, e 75 anos ou mais). Os coeficientes populacionais brutos de internações e de óbitos hospitalares foram calculados a partir das médias anuais do triênio por 10.000 habitantes e por 1.000 .000 de habitantes com base na população residente por sexo do Censo Demográfico nacional de $2000^{10}$. A padronização por idade utilizou o método direto considerando a população brasileira em 2000 total de ambos os sexos segundo os 5 intervalos selecionados. A letalidade foi obtida pela divisão dos coeficientes padronizados de óbitos hospitalares pelos coeficientes padronizados de internações de acordo com a faixa etária, sexo e/ou região. A média de permanência foi calculada dividindo-se o número total de dias de hospitalização pelo número de internações. O gasto médio por internação inclui despesas em enfermaria e com tratamento intensivo. Os valores pagos em reais por hospitalização foram convertidos pelo dólar norte-americano do último dia do mês seguinte ao de competência, já disponíveis no banco de dados do Ministério da Saúde (SIH/SUS). A taxa média de câmbio dos 36 meses equivaleu a 1 US $\$=2,0131$ 
reais, com variação de $17 \%$ entre o primeiro e o último mês.

Em relação aos procedimentos estatísticos, todos os intervalos de confiança são de 95\%. Para os coeficientes brutos e padronizados, o erro padrão dos intervalos de confiança foi obtido dividindo-se o numerador pela raiz quadrada das médias anuais de hospitalizações ou de óbitos conforme o caso ${ }^{11}$. Regressão logística múltipla foi desenvolvida para verificar associações com a ocorrência de óbito hospitalar. A análise dos dados foi realizada em SPSS® versão 10.0.1.

Quanto aos aspectos éticos, a fonte dos dados foram arquivos do SIH/SUS de domínio público, disponíveis na internet (www.datasus.gov.br) e divulgados de forma a preservar a identificação dos sujeitos, garantindo sua confidencialidade ${ }^{8}$.

\section{Resultados}

$\mathrm{Na}$ Tabela 1 encontram-se as características gerais das hospitalizações estudadas, cuja taxa bruta de óbitos atingiu 5,4\%. Nas hospitalizações, a média da idade dos homens (53,2 anos IC 95\% 53,1-53,3) foi significativamente menor do que a das mulheres (57,3 IC 95\% 57,3-57,4). Eles permaneceram mais tempo internados $(6,7$ dias IC 95\% 6,7-6,8) do que as pacientes do sexo feminino (6,3 dias IC 95\% 6,2-6,3), e suas hospitalizações foram mais dispendiosas (US\$ 155,36 IC 95\% 154,10-156,61) quando comparadas às das mulheres (US\$ 147,67 IC 95\% 146,80-148,54).

Na Tabela 2 observam-se as distribuições regionais das hospitalizações e dos óbitos hospitalares, ajustados por faixa etária, e a letalidade regional por sexo. As internações do sexo feminino $(7,49 /$ 10.000hab.) superam as do sexo masculino $(5,20 / 10.000$ hab.) em $44 \%$. Há maior diversidade entre as regiões quanto às internações das mulheres (coeficiente de variação (CV) 21,9\%) em relação às dos homens (CV 16,2\%). À semelhança das internações, os óbitos hospitalares femininos (38,1/1.000.000hab.) predominam sobre os masculinos (30,7/1.000.000hab.) em 24\%. Observa-se maior variação entre as regiões quanto à mortalidade hospitalar de homens (CV 21,2\%) do que de mulheres (CV 17,1\%).

Ao contrário das hospitalizações e da mortalidade hospitalar, a letalidade é menor para as mulheres $(5,0 \%)$ em relação aos homens (5,9\%). Apresenta maior variação regional para o sexo feminino (CV 32,4\%) do que para o masculino (CV 26,1\%), ambas maiores que as de hospitalização e de mortalidade hospitalar. A letalidade é maior para ambos os sexos no Nordeste e no Sudeste e menor na região Sul.

Na Tabela 3 encontra-se a distribuição regional das hospitalizações, dos óbitos hospitalares e da letalidade por faixa etária e sexo. O incremento das hospitalizações com o aumento da idade é pronunciado particularmente a partir dos 45 anos (coeficientes $>10 / 10.000$ hab.), sendo mais acentuado no sexo feminino. As hospitalizações das mulheres são mais freqüentes em todas as faixas etárias, mesmo analisadas por regiões, e apresentam maior variação regional considerando intervalos etários idênticos.

Observa-se o aumento da mortalidade com a progressão da idade, acentuado a partir dos 65 anos (coeficientes > 100/ 1.000.000hab.). No intervalo de 20-44 anos, os homens apresentam coeficientes de mortalidade mais elevados do que os das mulheres, invertendo a situação a partir dos 45 anos. Entretanto, há diferenças de mais de $50 \%$ nas mesmas faixas etárias em diferentes regiões. Em relação à letalidade, o sexo masculino destaca-se em todas as faixas etárias adultas, porém com diferenças entre as regiões de até mais de $80 \%$.

A Tabela 4 apresenta o gasto total médio por hospitalização, segundo a ocorrência ou não de óbito, e o gasto por 10.000hab. O valor médio por internação com óbito é sempre superior ao do sem óbito em todas as regiões e intervalos etários, sobretudo entre os mais jovens. Há grande variação inter-regional ( $\mathrm{CV}$ até $57 \%$ ) e intergeracional (CV até $89 \%$ ) do gas- 
Tabela 1 - Características gerais das hospitalizações por diabetes mellitus como diagnóstico principal (1) na rede pública, Brasil, 1999-2001

Table 1 - National health system (SUS) hospitalizations for diabetes mellitus as first-listed diagnosis, general characteristics, Brazil, 1999-2001

\begin{tabular}{|c|c|c|c|c|c|c|}
\hline & \multicolumn{2}{|c|}{ Total } & \multicolumn{2}{|c|}{ Com óbito } & \multicolumn{2}{|c|}{ Sem óbito } \\
\hline & N & (\% ou IC $95 \%)$ & $\mathrm{N}$ & (\% ou IC $95 \%)$ & $\mathrm{N}$ & (\% ou IC 95\%) \\
\hline Hospitalizações & 327.800 & $(100,0)$ & 17.760 & $(5,4)$ & 310.040 & $(94,6)$ \\
\hline \multicolumn{7}{|l|}{ Sexo } \\
\hline Masculino & 124.703 & $(100,0)$ & 7.199 & $(5,8)$ & 117.504 & $(94,2)$ \\
\hline Feminino & 203.097 & $(100,0)$ & 10.561 & $(5,2)$ & 192.536 & $(94,8)$ \\
\hline Idade - anos & 55,8 & $(55,7-55,8)$ & 63,8 & $(63,5-64,0)$ & 55,3 & $(55,1-55,5)$ \\
\hline Masculino & 53,2 & $(53,1-53,3)$ & 61,1 & $(60,8-61,5)$ & 52,7 & $(52,3-53,1)$ \\
\hline Feminino & 57,3 & $(57,3-57,4)$ & 65,6 & $(65,3-65,9)$ & 56,9 & $(56,6-57,2)$ \\
\hline \multicolumn{7}{|l|}{ Faixas etárias } \\
\hline \multicolumn{7}{|l|}{ Masculino } \\
\hline $0-19$ & 8.213 & $(100,0)$ & 85 & $(1,0)$ & 8.128 & $(99,0)$ \\
\hline $20-44$ & 27.610 & $(100,0)$ & 1.121 & $(4,1)$ & 26.489 & $(95,9)$ \\
\hline $45-64$ & 51.139 & $(100,0)$ & 2.614 & $(5,1)$ & 48.525 & $(94,9)$ \\
\hline $65-74$ & 23.328 & $(100,0)$ & 1.743 & $(7,5)$ & 21.585 & $(92,5)$ \\
\hline $75+$ & 14.413 & $(100,0)$ & 1.636 & $(11,4)$ & 12.777 & $(88,6)$ \\
\hline \multicolumn{7}{|l|}{ Feminino } \\
\hline $0-19$ & 11.089 & $(100,0)$ & 164 & $(1,5)$ & 10.925 & $(98,5)$ \\
\hline $20-44$ & 29.459 & $(100,0)$ & 856 & $(2,9)$ & 28.603 & $(97,1)$ \\
\hline $45-64$ & 82.494 & $(100,0)$ & 3.273 & $(4,0)$ & 79.221 & $(96,0)$ \\
\hline $65-74$ & 48.195 & $(100,0)$ & 2.955 & $(6,1)$ & 45.240 & $(93,9)$ \\
\hline $75+$ & 31.860 & $(100,0)$ & 3.313 & $(10,4)$ & 28.547 & $(89,6)$ \\
\hline Média de permanência - dias & 6,4 & $(6,4-6,5)$ & 6,5 & $(6,3-6,6)$ & 6,4 & $(6,3-6,6)$ \\
\hline Masculino & 6,7 & $(6,7-6,8)$ & 6,6 & $(6,3-6,9)$ & 6,7 & $(6,4-7,0)$ \\
\hline Feminino & 6,3 & $(6,2-6,3)$ & 6,4 & $(6,2-6,6)$ & 6,3 & $(6,1-6,5)$ \\
\hline Gasto por internação - US\$ & 150,59 & $(149,87-151,32)$ & 275,27 & $(268,37-282,16)$ & 143,45 & $(136,56-150,35)$ \\
\hline Masculino & 155,36 & $(154,10-156,61)$ & 274,26 & $(263,86-284,66)$ & 148,07 & $(137,68-158,47)$ \\
\hline Feminino & 147,67 & $(146,80-148,54)$ & 275,95 & $(266,77-285,13)$ & 140,63 & $(131,46-149,81)$ \\
\hline \multicolumn{7}{|l|}{ Região de residência } \\
\hline \multicolumn{7}{|l|}{ Masculino } \\
\hline Norte & 5.927 & $(100,0)$ & 313 & $(5,3)$ & 5.614 & $(94,7)$ \\
\hline Nordeste & 28.349 & $(100,0)$ & 2.016 & $(7,1)$ & 26.333 & $(92,9)$ \\
\hline Sudeste & 59.394 & $(100,0)$ & 3.714 & $(6,3)$ & 55.680 & $(93,7)$ \\
\hline Sul & 21.499 & $(100,0)$ & 785 & $(3,7)$ & 20.714 & $(96,3)$ \\
\hline Centro-Oeste & 9.534 & $(100,0)$ & 371 & $(3,9)$ & 9.163 & $(96,1)$ \\
\hline \multicolumn{7}{|l|}{ Feminino } \\
\hline Norte & 8.583 & $(100,0)$ & 380 & $(4,4)$ & 8.203 & $(95,6)$ \\
\hline Nordeste & 46.289 & $(100,0)$ & 3.075 & $(6,6)$ & 43.214 & $(93,4)$ \\
\hline Sudeste & 91.867 & $(100,0)$ & 5.277 & $(5,7)$ & 86.590 & $(94,3)$ \\
\hline Sul & 41.270 & $(100,0)$ & 1.286 & $(3,1)$ & 39.984 & $(96,9)$ \\
\hline Centro-Oeste & 15.088 & $(100,0)$ & 543 & $(3,6)$ & 14.545 & $(96,4)$ \\
\hline
\end{tabular}

Nota: IC 95\% - intervalo de confiança de $95 \%$.

(1) diagnóstico principal CID-10 E10-E14 combinado a procedimento realizado. 
Tabela 2 - Hospitalizações, óbitos hospitalares e letalidade hospitalar anuais por diabetes mellitus como diagnóstico principal (1) na rede pública segundo região e sexo, padronizados por idade, Brasil, 1999-2001

Table 2 - National health system (SUS) hospitalizations for diabetes mellitus as first-listed diagnosis(admissions/10.000, deaths/1.000.000 and lethality) by geographic region and sex, standardized by age, Brazil, 1999-2001

\begin{tabular}{|c|c|c|c|}
\hline \multicolumn{4}{|c|}{ Hospitalizações por 10.000 habitantes (IC 95\%) (2) } \\
\hline & Masculino & Feminino & Total \\
\hline \multicolumn{4}{|l|}{ Região } \\
\hline Norte & $4,17(3,99-4,36)$ & $6,29(6,06-6,52)$ & $5,21(5,06-5,36)$ \\
\hline Nordeste & $4,47(4,38-4,56)$ & $6,39(6,29-6,50)$ & $5,51(5,44-5,58)$ \\
\hline Sudeste & $5,52(5,44-5,59)$ & $7,34(7,26-7,43)$ & $6,52(6,46-6,58)$ \\
\hline Sul & $5,72(5,59-5,85)$ & $9,64(9,48-9,80)$ & $7,82(7,72-7,93)$ \\
\hline Centro-Oeste & $6,13(5,92-6,35)$ & $9,84(9,57-10,11)$ & $8,01(7,84-8,18)$ \\
\hline Total & $5,20(5,15-5,25)$ & $7,49(7,44-7,55)$ & $6,44(6,40-6,47)$ \\
\hline \multicolumn{4}{|c|}{ Óbitos hospitalares por 1.000 .000 de habitantes (IC 95\%) (2) } \\
\hline & Masculino & Feminino & Total \\
\hline \multicolumn{4}{|l|}{ Região } \\
\hline Norte & $23,0(18,6-27,4)$ & $28,7(23,7-33,7)$ & $25,8(22,5-29,1)$ \\
\hline Nordeste & $31,7(29,3-34,0)$ & $41,2(38,7-43,7)$ & $36,8(35,1-38,6)$ \\
\hline Sudeste & $35,2(33,3-37,2)$ & $40,5(38,6-42,4)$ & $38,4(37,0-39,8)$ \\
\hline Sul & $21,7(19,1-24,3)$ & $29,2(26,4-31,9)$ & $25,9(23,9-27,8)$ \\
\hline Centro-Oeste & $25,5(21,0-30,0)$ & $37,5(32,0-42,9)$ & $31,7(28,1-35,2)$ \\
\hline Total & $30,7(29,5-32,0)$ & $38,1(36,8-39,3)$ & $34,9(34,0-35,8)$ \\
\hline \multicolumn{4}{|c|}{ Letalidade hospitalar (\%) (3) } \\
\hline & Masculino & Feminino & Total \\
\hline \multicolumn{4}{|l|}{ Região } \\
\hline Norte & 5,5 & 3,9 & 5,0 \\
\hline Nordeste & 7,1 & 6,1 & 6,7 \\
\hline Sudeste & 6,4 & 5,3 & 5,9 \\
\hline Sul & 3,8 & 2,8 & 3,3 \\
\hline Centro-Oeste & 4,2 & 3,3 & 4,0 \\
\hline Total & 5,9 & 5,0 & 5,4 \\
\hline
\end{tabular}

to populacional.

$\mathrm{Na}$ Tabela 5 encontram-se os resultados de um modelo por regressão logística múltipla para a ocorrência ou não de óbito hospitalar considerando as variáveis sexo, faixa etária, gasto por internação e região de residência do paciente. A razão de chances de um homem falecer em uma hospitalização por DM é $20 \%$ maior que a de uma mulher. A idade acima de 75 anos praticamente triplica a razão de chances de ocorrência de óbito em relação ao intervalo 45-64 anos, por sua vez quatro ve- zes o de 0-19 anos. Os habitantes das regiões Nordeste e Sudeste apresentaram razão de chances duas vezes maior de morrer durante uma internação que os da região Sul.

\section{Discussão}

Estima-se que, nos próximos vinte anos, o número de casos de diabetes aumentará em até $170 \%$ em países em desenvolvimento, como o Brasil, contrastando com o acréscimo de $42 \%$ em países desen- 
Tabela 3 - Hospitalizações, óbitos hospitalares e letalidade anuais por diabetes mellitus comodiagnóstico principal (1) na rede pública segundo sexo, faixa etária e região, Brasil, 19992001

Table 3 - National health system (SUS) hospitalizations for diabetes mellitus as first-listed diagnosis (admissions/10.000, deaths/1.000.000 and lethality) by sex, age and geographicregion, Brazil, 1999-2001

\begin{tabular}{|c|c|c|c|c|c|c|}
\hline \multicolumn{7}{|c|}{ Hospitalizações por 10.000 habitantes } \\
\hline & Norte & Nordeste & Sudeste & Sul & C.Oeste & Total \\
\hline \multicolumn{7}{|c|}{ Masculino } \\
\hline $0-19$ & 0,2 & 0,4 & 1,1 & 1,1 & 1,1 & 0,8 \\
\hline $20-44$ & 1,7 & 2,1 & 3,4 & 2,8 & 3,7 & 2,8 \\
\hline $45-64$ & 11,5 & 12,4 & 14,2 & 14,7 & 15,8 & 13,8 \\
\hline $65-74$ & 27,8 & 25,5 & 26,0 & 31,4 & 30,1 & 27,1 \\
\hline $75+$ & 30,7 & 29,5 & 31,2 & 37,6 & 35,9 & 31,8 \\
\hline \multicolumn{7}{|c|}{ Feminino } \\
\hline $0-19$ & 0,3 & 0,6 & 1,5 & 1,5 & 1,4 & 1,1 \\
\hline $20-44$ & 1,7 & 2,1 & 3,1 & 4,2 & 3,6 & 2,9 \\
\hline $45-64$ & 19,9 & 18,5 & 19,3 & 24,7 & 26,4 & 20,4 \\
\hline $65-74$ & 43,3 & 42,8 & 42,5 & 60,4 & 63,1 & 46,5 \\
\hline $75+$ & 40,3 & 44,3 & 48,4 & 66,9 & 69,8 & 50,6 \\
\hline \multicolumn{7}{|c|}{ Óbitos hospitalares por 1.000 .000 de habitantes } \\
\hline & Norte & Nordeste & Sudeste & Sul & C.Oeste & Total \\
\hline \multicolumn{7}{|c|}{ Masculino } \\
\hline $0-19$ & 0,9 & 0,9 & 0,7 & 0,6 & 1,3 & 0,8 \\
\hline $20-44$ & 7,3 & 12,3 & 13,9 & 6,5 & 9,8 & 11,6 \\
\hline $45-64$ & 42,9 & 76,6 & 82,0 & 44,6 & 55,8 & 70,4 \\
\hline $65-74$ & 212,4 & 202,2 & 222,6 & 145,5 & 193,4 & 202,5 \\
\hline $75+$ & 251,3 & 344,0 & 414,5 & 316,2 & 260,8 & 360,7 \\
\hline \multicolumn{7}{|c|}{ Feminino } \\
\hline $0-19$ & 0,9 & 2,0 & 1,7 & 1,4 & 1,3 & 1,6 \\
\hline $20-44$ & 6,2 & 8,6 & 9,6 & 5,6 & 10,0 & 8,5 \\
\hline $45-64$ & 66,9 & 94,8 & 84,0 & 59,3 & 71,3 & 81,1 \\
\hline $65-74$ & 253,8 & 316,0 & 290,5 & 220,8 & 308,6 & 285,1 \\
\hline $75+$ & 298,0 & 511,3 & 587,5 & 433,5 & 505,4 & 525,9 \\
\hline \multicolumn{7}{|c|}{ Letalidade hospitalar (\%) (2) } \\
\hline & Norte & Nordeste & Sudeste & Sul & C.Oeste & Total \\
\hline \multicolumn{7}{|c|}{ Masculino } \\
\hline 0-19 & 4,7 & 2,1 & 0,7 & 0,6 & 1,2 & 1,0 \\
\hline $20-44$ & 4,4 & 5,8 & 4,1 & 2,3 & 2,7 & 4,1 \\
\hline $45-64$ & 3,7 & 6,2 & 5,8 & 3,0 & 3,5 & 5,1 \\
\hline $65-74$ & 7,6 & 7,9 & 8,5 & 4,6 & 6,4 & 7,5 \\
\hline $75+$ & 8,2 & 11,6 & 13,3 & 8,4 & 7,3 & 11,4 \\
\hline \multicolumn{7}{|c|}{ Feminino } \\
\hline $0-19$ & 2,6 & 3,5 & 1,1 & 0,9 & 0,9 & 1,5 \\
\hline $20-44$ & 3,6 & 4,1 & 3,1 & 1,3 & 2,7 & 2,9 \\
\hline $45-64$ & 3,4 & 5,1 & 4,3 & 2,4 & 2,7 & 4,0 \\
\hline $65-74$ & 5,9 & 7,4 & 6,8 & 3,7 & 4,9 & 6,1 \\
\hline $75+$ & 7,4 & 11,5 & 12,1 & 6,5 & 7,2 & 10,4 \\
\hline
\end{tabular}

Nota: C.Oeste - região Centro-Oeste.

(1) diagnóstico principal CID-10 E10-E14 combinado a procedimento realizado.

(2) calculada pela divisão dos coeficientes de óbitos pelos de hospitalizações. 
Tabela 4 - Gasto (US\$) por internação por diabetes mellitus (conforme ocorrência ou não de óbito) como diagnóstico principal (1) na rede pública e gasto anual (US\$) por 10.000 habitantes, por faixa etária e região, Brasil, 1999-2001

Table 4 - National health system (SUS) hospitalizations for diabetes mellitus as first-listed diagnosis (expenditures (US\$)/ admission by death occurrence or not and expenditures (US\$)/10.000) by age and geographic region, Brazil, 1999-2001

\begin{tabular}{|c|c|c|c|c|c|c|}
\hline \multicolumn{7}{|c|}{ Gasto (US\$) por internação } \\
\hline & Norte & Nordeste & Sudeste & Sul & C.Oeste & Total \\
\hline \multicolumn{7}{|c|}{ Com óbito } \\
\hline 0-19 & 256,76 & 437,84 & 631,08 & 339,74 & 760,94 & 510,75 \\
\hline $20-44$ & 261,48 & 256,25 & 373,71 & 341,38 & 503,14 & 342,84 \\
\hline $45-64$ & 276,19 & 227,76 & 297,86 & 357,48 & 444,96 & 291,61 \\
\hline $65-74$ & 307,39 & 214,31 & 255,74 & 303,81 & 366,25 & 258,18 \\
\hline $75+$ & 207,33 & 214,95 & 228,93 & 254,56 & 377,04 & 233,20 \\
\hline \multirow[t]{2}{*}{ Total } & 269,63 & 227,36 & 279,67 & 310,04 & 424,28 & 275,27 \\
\hline & Norte & Nordeste & Sudeste & Sul & C.Oeste & Total \\
\hline \multicolumn{7}{|c|}{ Sem óbito } \\
\hline $0-19$ & 160,92 & 196,05 & 196,39 & 214,43 & 184,02 & 197,72 \\
\hline $20-44$ & 126,28 & 143,15 & 161,86 & 145,96 & 145,71 & 152,42 \\
\hline $45-64$ & 122,55 & 131,67 & 141,98 & 133,66 & 136,81 & 136,69 \\
\hline $65-74$ & 128,89 & 134,27 & 141,39 & 133,11 & 139,72 & 137,27 \\
\hline $75+$ & 124,54 & 132,49 & 141,31 & 137,10 & 141,24 & 137,40 \\
\hline Total & 126,13 & 137,03 & 149,42 & 140,86 & 143,19 & 143,45 \\
\hline \multicolumn{7}{|c|}{ Gasto anual (US\$) por 10.000 habitantes } \\
\hline & Norte & Nordeste & Sudeste & Sul & C.Oeste & Total \\
\hline $0-19$ & 43,25 & 102,51 & 256,83 & 278,88 & 235,21 & 190,33 \\
\hline $20-44$ & 222,92 & 315,23 & 544,18 & 518,99 & 568,39 & 459,53 \\
\hline $45-64$ & $1.993,17$ & $2.142,52$ & $2.523,77$ & $2.768,73$ & $3.083,10$ & $2.474,88$ \\
\hline $65-74$ & $4.972,51$ & $4.901,16$ & $5.275,56$ & $6.617,04$ & $7.084,38$ & $5.474,80$ \\
\hline $75+$ & $4.682,30$ & $5.354,31$ & $6.336,32$ & $8.019,88$ & $8.530,11$ & $6.305,50$ \\
\hline Total & 498,57 & 746,20 & $1.094,34$ & $1.220,38$ & $1.083,52$ & 969,09 \\
\hline
\end{tabular}

Nota: C.Oeste - região Centro-Oeste.

(1) diagnóstico principal CID-10 E10-E14 combinado a procedimento realizado.

volvidos $^{12}$. O impacto econômico do diabetes é expressivo, e as hospitalizações consomem parcela importante dos recursos da sociedade. Representam 55\% dos custos diretos do DM tipo 2 na Europa ${ }^{13}$, $44 \%$ nos EUA $^{14}$ e $10 \%$ na América Latina ${ }^{15}$.

Estudos sobre internações hospitalares em nível nacional usualmente apresentam dificuldades em razão das diferentes formas de registros nos prontuários. Nos EUA, o Centro Nacional de Estatísticas de Saúde (NCHS) conduz anualmente a "National Hospital Discharge Survey" (NHDS) a partir de dados de hospitais nãofederais destinados à curta permanência desde $1965^{16}$. No Brasil, o preenchimento obrigatório do diagnóstico principal em um documento único para autorização da internação hospitalar (AIH) na rede pública, de cobertura universal, ofereceu a possibilidade de estudos de morbidade hospitalar através do Sistema de Informações Hospitalares do Sistema Único de Saúde (SIH/SUS).

No SIH/SUS, constatamos que as internações com DM como diagnóstico principal representaram 6,4 hospitalizações/ 10.000hab. Nos EUA, as internações por diabetes (CID-9 código 250) como motivo principal de internação ("first-listed") atingiram 20,0/10.000hab. em 2000 ${ }^{16}$. Caso o Brasil apresentasse a estrutura etária nor- 
Tabela 5 - Variáveis associadas por regressão logística múltipla com óbitos hospitalares em 327.800 hospitalizações por diabetes mellitus como diagnóstico principal (1) na rede pública, Brasil, 1999-2001

Table 5 - National health system (SUS) hospitalizations ( $n=327,800)$ for diabetes mellitus as firstlisted diagnosis, multiple logistic regression of factors associated with hospitalar death, Brazil, 1999-2001.

\begin{tabular}{lll}
\hline Variável & OR ajustado & $($ IC 95\%) \\
\hline $\begin{array}{l}\text { Sexo } \\
\text { Feminino }\end{array}$ & 1,00 & \\
Masculino & 1,21 & $(1,17-1,24)$ \\
& & \\
Idade & 1,00 & \\
$0-19$ anos & 3,19 & $(2,78-3,67)$ \\
$20-44$ anos & 4,43 & $(3,88-5,06)$ \\
$45-64$ anos & 6,89 & $(6,03-7,89)$ \\
65-74 anos & 11,73 & $(10,25-13,41)$ \\
$75+a n o s$ & & \\
Gasto & 1,18 & $(1,17-1,19)$ \\
A cada 100 US\$ & & \\
Região de residência & 1,00 & $(1,40-1,67)$ \\
Sul & 1,53 & $(1,99-2,21)$ \\
Norte & 2,09 & $(1,78-1,97)$ \\
Nordeste & 1,87 & $(1,09-1,28)$ \\
Sudeste & 1,18 & \\
Centro-Oeste & $95 \%)$ & \\
\hline
\end{tabular}

OR (IC 95\%) - odds ratio (intervalo de confiança de 95\%).

(1) diagnóstico principal CID-10 E10-E14 combinado a procedimento realizado.

te-americana ${ }^{17}$, as internações por DM passariam a 10,2/10.000hab., elevando-se de um terço para a metade do coeficiente dos EUA. Admitindo-se que a cobertura da rede pública represente $70 \%$ de todas as internações do sistema de saúde brasileiro $^{18}$, chegaria a quase três quartos da situação norte-americana.

O impacto do diabetes vem sendo identificado sob diferentes aspectos no Brasil. O estudo mais completo sobre a prevalência de DM foi realizado entre 1986-88, em amostras representativas da população adulta em comunidades de nove capitais, que estimou em 7,6\% ajustado para a população do Censo Demográfico de $1980^{4}$. Entre as causas de "carga de doença”, o DM situou-se em primeiro lugar para ambos os sexos em 1998 através da metodologia DALY ("Disability Adjusted Life
Years" - Anos de Vida Ajustados por Incapacidade $)^{19}$. Um diagnóstico da situação de saúde da população idosa ( $\geq 60$ anos) com dados do SIH/SUS encontrou o DM (CID9 código 250) em $12^{\circ}$ lugar como motivo principal de internação hospitalar entre homens $\left(1,7 \%\right.$ do total) e em $6^{\circ}$ entre mulheres $\left(3,5 \%\right.$ do total) em $1996^{20}$.

A conjunção desses dados delineia um quadro importante. Já no estudo multicêntrico sobre a prevalência do DM no Brasil afirmava-se que a situação era comparável à de países como EUA, Itália, Israel e Argentina, particularmente em cidades de regiões mais desenvolvidas, como São Paulo $(9,7 \%)$ no Sudeste, ou Porto Alegre $(8,9 \%)$ na região $\mathrm{Sul}^{4}$. Parte das conseqüências desse perfil populacional reflete-se no consumo dos serviços hospitalares, mesmo considerando-se que analisa- 
mos apenas o "diagnóstico principal” da internação, não computando as demais hospitalizações atribuíveis ao DM. Revela, portanto, somente parte do "iceberg epidemiológico".

A distribuição entre os sexos das hospitalizações e dos óbitos hospitalares por DM no Brasil denota diferenças importantes de utilização dos serviços e enseja abordagens específicas das políticas de saúde. Nos EUA, as hospitalizações por DM como primeiro diagnóstico são semelhantes entre mulheres (19,6/10.000hab.) e homens $(20,5 / 10.000 h a b .)^{16}$. No Brasil, a diferença atinge $50 \%$ se ajustarmos para as faixas etárias norte-americanas correspondentes, passando o sexo feminino de 7,5 para 12/10.000hab., e o masculino, de 5,2 para 8/10.000hab.

O predomínio feminino na utilização de serviços de saúde é bastante conhecido. Na Itália, as mulheres apresentaram taxas mais elevadas de hospitalização por DM tipo 2 do que os homens ${ }^{21}$, o que também constatamos em relação aos óbitos hospitalares (tabelas 1 a 3). Entretanto, naquele país, a diferença foi mais evidente abaixo dos 65 anos, enquanto no Brasil ela ocorreu acima dessa idade.

A predominância das hospitalizações femininas é reforçada na comparação com os dados de prevalência do DM. Apesar de não haver diferenças entre homens $(7,5 \%)$ e mulheres $(7,6 \%)$ no Estudo Multicêntrico sobre a Prevalência de DM no Brasil, as mulheres conheciam mais sua condição $(4,5 \%)$ do que os homens $(3,1 \%)$, o mesmo ocorrendo com pessoas entre 60-69 anos $(9,9 \%)$ em relação às de $30-39$ anos $(1,2 \%)^{4}$. Há implicações óbvias na demanda dos serviços de saúde que, possivelmente, repercutam na maior utilização de recursos hospitalares por mulheres com mais idade. Cabe mencionar que o estudo multicêntrico excluiu as gestantes, e este trabalho, as hospitalizações por DM gestacional.

É possível que haja "filtros" importantes no acesso dos homens ao atendimento hospitalar especificamente por DM. Na Pesquisa Nacional por Amostra de Domicílios
(PNAD) de 1998, as mulheres utilizaram mais os serviços de saúde de um modo geral, mesmo quando controlado o efeito da restrição ou não de atividades por motivo de saúde ${ }^{22}$. Esse fenômeno pode estar atuando com mais intensidade nas regiões Sul e Centro-Oeste, particularmente em pacientes acima dos 65 anos (Tabela 3), que apresentam os maiores coeficientes de internação e diferenciais entre os sexos (cerca de 2 vezes). Nos EUA, idade igual ou superior a 75 anos e sexo feminino estiveram associados a maior probabilidade de internações potencialmente evitáveis de beneficiários do Medicare com DM tipo $2^{23}$.

O DM atinge todos os grupos etários, mas se torna mais prevalente e tem maiores efeitos sobre o consumo de serviços de saúde entre pessoas com mais idade ${ }^{24}$. Estima-se que, até 2025, o aumento do número de casos de diabetes em países em desenvolvimento dar-se-á mais no intervalo de 45-64 anos, contrastando com os países desenvolvidos, onde o incremento se concentra acima dos 65 anos $^{12}$.

Os resultados brasileiros aproximamse dos observados em países desenvolvidos. Apesar do número absoluto maior de internações entre 45-64 anos (41\%), os coeficientes populacionais a partir dos 65 anos são 2 vezes maiores que os da faixa imediatamente anterior. Nos EUA, as internações por DM como motivo principal em 2000 atingiram 33,9 e 55,7/10.000 hab., respectivamente, nos intervalos de 45-64 e 65 anos ou mais ${ }^{16}$. No Brasil, obtivemos 17,2 e 39,5/10.00hab., respectivamente. Enquanto o valor da primeira faixa etária corresponde a $50 \%$ da taxa dos norte-americanos, a última já equivale a $71 \%$. Na região Sul, uma das mais desenvolvidas do país, o coeficiente de 65 anos ou mais corresponde a $90 \%$ dos EUA - somente considerando as internações na rede pública.

O incremento das hospitalizações com a idade é mais intenso que o da prevalência. O Estudo Multicêntrico sobre a Prevalência de DM no Brasil encontrou 6,5 vezes mais diabéticos na faixa etária de 60 - 
69 anos $(17,4 \%)$ em relação à de 30-39 anos $(2,7 \%)^{4}$. Utilizando os mesmos intervalos, a relação nas hospitalizações/10.000hab. é de 10,2 vezes, sugerindo dificuldade de acesso do estrato mais jovem, excesso de internações na faixa etária mais elevada e/ ou reflexo das complicações decorrentes da duração da doença.

Há diversidade regional de intensidade moderada no volume de hospitalizações (CV máximo 22\% e quociente entre os coeficientes maiores e menores de até $1,6 \mathrm{vez})$. Variações regionais mais expressivas (> 2,5 vezes) em hospitalizações por DM foram encontradas utilizando-se como denominador tanto a população de diabéticos ${ }^{25}$ quanto a população geral ${ }^{26}$.

Quanto aos óbitos hospitalares, eles podem medir tanto o processo de cuidado do paciente quanto o resultado ${ }^{27}$. Constatamos que mulheres internam e morrem mais do que homens em termos de coeficientes populacionais, porém como menor proporção em todas as regiões. A predominância da letalidade masculina se mantém, mesmo comparando-se pontos extremos. É maior que a do sexo feminino na região Nordeste, que apresenta os maiores coeficientes de mortalidade e de letalidade femininos do país na maioria das faixas etárias, e na região Sul, que apresenta os menores indicadores para o sexo masculino. À semelhança do constatado em diversos países, a idade também é um preditor independente da mortalidade por DM do tipo 2 no Brasil ${ }^{28}$. Óbito hospitalar e letalidade, em ambos os sexos, progridem consistentemente com a idade. Acima dos 45 anos, são maiores nas regiões Nordeste e Sudeste, situadas paradoxalmente no espectro oposto em termos de desenvolvimento.

A progressão da idade também afeta a distribuição dos óbitos hospitalares entre os sexos. Nossos resultados foram consistentes com dois estudos sobre mortalidade por DM realizados a partir de declarações do óbito. No Estado de São Paulo, a análise dos óbitos de 1992 com menção a DM evidenciou que o sexo masculino (12/ 1.000.000hab.) predominava sobre o feminino (9/1.000.000hab.) abaixo dos 40 anos, enquanto o indicador das mulheres (724/ 1.000.000hab.) era maior que o dos homens (504/1.000.000hab.) na faixa etária superior $^{29}$. No Brasil, o coeficiente de mortalidade por complicações agudas do DM entre 1996-2002 não apresentou diferenças significativas entre os sexos abaixo dos 10 anos de idade, foi maior no sexo feminino entre 10-19 anos, equivalente entre 20-29 anos, maior no sexo masculino entre 30-49 e maior no sexo feminino a partir dos $50 \operatorname{anos}^{30}$.

Em relação ao tempo de permanência, a média brasileira (6,4 dias IC 95\% 6,4-6,5) é superior à norte-americana (5,2 IC 95\% $5,1-5,2$ ) correspondente ao mesmo triênio calculada a partir dos dados do Centers for Disease Control and Prevention ${ }^{31}$. Há também pouca variação (CV 5,5\%) entre as regiões brasileiras. Entre os hospitais distritais da Finlândia, oscila de 4,9 a 10,7 dias ${ }^{25}$ enquanto no Brasil varia de 5,9 (IC 95\% 5,9$6,0)$ no Sul a $6,7(6,6-6,8)$ no Nordeste. É possível que a menor variabilidade das médias brasileiras deva-se ao caráter de pagamento prospectivo por DRG (grupo de diagnósticos relacionados) do SIH/SUS.

Em nosso estudo, a permanência hospitalar não está associada à letalidade. As médias de dias de internação com e sem ocorrência de óbito para o conjunto do país não diferem significativamente, apesar de algumas particularidades regionais. As internações com óbito nas regiões $\mathrm{Su}$ deste $(7,2$ IC 95\% 7,0-7,5) e Centro-Oeste $(7,2$ IC 95\% 6,5-8,0) são mais prolongadas do que as sem óbito (6,6 IC 95\% 6,5-6,6 e 6,0 IC 95\% 5,8-6,1), enquanto no Nordeste ocorre o contrário (5,5 IC 95\% 5,2-5,7 e 6,8 IC 95\% 6,7-6,9). Apesar da significância estatística, tais resultados parecem à primeira vista pouco relevantes, tanto clinica quanto administrativamente, por implicarem em diferenças de apenas uma diária hospitalar. Contudo, talvez sejam importantes sob a ótica macrossetorial de ocupação de leitos e alocação de recursos.

À semelhança de outros autores ${ }^{24}$, verificamos que as internações prolongadas 
estão associadas a gastos médios maiores $\left(r^{2}=0,073 ; p<0,01\right)$. Ainda assim, o valor médio pago por uma internação foi de US\$ 150,59 (IC 95\% 149,87-151,32), que corresponde a $69 \%$ da média das internações do SUS no período. Estudo exploratório no mesmo sistema já demonstrara que as hospitalizações do grupo III (doenças das glândulas endócrinas, da nutrição, do metabolismo e transtornos imunitários) da CID-9 correspondiam a $75 \%$ em $1991^{32}$.

As internações com óbito são mais dispendiosas do que as sem óbito, ainda que apresentem a mesma média de permanência. Tal diferença refere-se principalmente a despesas em unidades de tratamento intensivo, em média 6,5 vezes maiores quando ocorre óbito (US\$ 61,00), e a serviços laboratoriais, indicando maior intensidade de utilização tecnológica. Os valores mais elevados encontrados em jovens ( $<20$ anos) provavelmente se devem a hospitalizações por DM tipo 1. Em relação à distribuição regional, as internações com óbitos do Centro-Oeste são sistematicamente mais dispendiosas que as demais e apresentam as maiores diferenças em relação às sem óbitos. A média de despesas com tratamento intensivo em caso de morte é 2 vezes maior que a nacional e representa $30 \%$ do valor total, enquanto em outras regiões varia de $18 \%$ a $26 \%$, o que certamente reflete aspectos de qualidade assistencial e processo decisório clínico distintos daqueles do restante do país.

Uma forma diferenciada de analisar o gasto em saúde é examiná-lo sob a ótica da incidência do gasto por grupo populacional. Sabe-se que indivíduos acima de 65 anos respondem por aproximadamente 2/ 3 de todos custos médicos relacionados ao diabetes $^{33}$. Em nossos dados, ainda que sob o enfoque de gastos e não de custos, essa faixa etária respondeu por $35 \%$ dos gastos, enquanto a de 45-64 anos respondeu por $39 \%$. Entretanto, deve ser considerado que estudamos apenas hospitalizações públicas com diagnóstico principal por DM.

O gasto total por 10.000 habitantes pode evidenciar as transferências orçamentári- as entre gerações ou regiões nas hospitalizações por DM. Observamos que acima dos 65 anos representa de 6 a 10 vezes o valor médio por grupo de 10.000 hab. na mesma região. Os habitantes das regiões Sul, Sudeste e Centro-Oeste (as mais desenvolvidas) apresentam maior gasto per capita do que os do Norte e Nordeste, refletindo não apenas maior incidência de hospitalizações (Tabela 1), como também valores médios por internação mais elevados, seja com ou sem óbito (Tabela 4). A maior letalidade nas regiões Nordeste e Sudeste (Tabela 2) e o menor coeficiente de mortalidade por complicações agudas do DM, entre 1996-2002, encontrado na região $\mathrm{Sul}^{30}$ evidenciam desigualdades importantes na utilização dos serviços de saúde.

Algumas limitações de nosso estudo devem ser mencionadas. A fonte dos dados (o SIH/SUS) foi desenvolvida inicialmente para funções administrativo-financeiras, com o propósito de cobrança, e pode não estar isenta de erros de codificação, intencionais ou não, ou de diagnóstico. O SIH/SUS paga de forma parcialmente prospectiva, permitindo aos prestadores de serviços conhecer os diagnósticos mais vantajosos e, assim, mudar a informação apresentada ou evitar a internação de pacientes com determinadas características ${ }^{34}$. Nossa análise restringiu-se somente ao diagnóstico principal, tal como apresentado ao SIH/SUS; as hospitalizações atribuíveis ao DM serão objeto de outra investigação. Estimativas baseadas apenas no diagnóstico principal não capturam toda a magnitude do impacto do diabetes mellitus na sociedade. Seria necessário utilizar metodologia diversa, como a do risco atribuível, para melhor dimensionar a carga da doença. Além disto, os dados referem-se apenas às internações de estabelecimentos próprios ou contratados pela rede pública (SUS), ainda que representem cerca de $70 \%$ das hospitalizações do país. Por último, o SIH/SUS não incorpora variáveis críticas com potencial explicativo para diferenças regionais ou ocorrência de óbito, como índice de massa corporal, raça, escolaridade, gravidade da condição clínica no mo- 
mento da internação, grau de utilização dos serviços, reinternações e outras. Os resultados aqui apresentados não permitem identificar o papel desses possíveis fatores, particularmente nas diferenças regionais.

Neste sentido, sugere-se a realização de pesquisas que incorporem variáveis não presentes no SIH/SUS e características populacionais. Há necessidade de estudos específicos de utilização dos serviços de saúde e das características dos pacientes hospitalizados na rede pública que permitam extrapolações regionais. Deveria também ser realizado um esforço para atualizar as estimativas de prevalência, sobretudo de maior abrangência geográfica do que aquelas obtidas no estudo multicêntrico dos anos $80^{4}$.

\section{Considerações finais}

Este trabalho apresentou, pela primeira vez, um panorama das internações por DM como diagnóstico principal no Sistema Único de Saúde. Apesar das limitações, os resultados reforçam tendências já identificadas em outros países. Observamos também importantes desigualdades regionais e de distribuição sexual nas hospitalizações.

Sabe-se que os determinantes sociais desempenham papel fundamental para as possíveis explicações de tais variações. A importância de fenômenos como a urbanização, o envelhecimento populacional, as transições epidemiológica e nutricional e as modificações de estilo de vida, interagindo com as condições socioeconômicas da população, a organização da produção e das classes sociais, os fatores etiológicos conhecidos e desconhecidos, a história natural prolongada e a multiplicidade de fatores de risco constituem um desafio para o enfrentamento do diabetes.

Apesar da complexidade da situação e da escassez de recursos, há uma necessidade clara de intervenção para reduzir iniqüidades. Acreditamos que, entre os instrumentos para essas ações de saúde pública, a difusão e a consolidação de estratégias preventivas, articuladas intra e intersetorialmente, possam prover cobertura mais adequada da população, evitar as hospitalizações e, sobretudo, reduzir o impacto psicossocial e econômico causado pelo próprio diabetes mellitus.

\section{Agradecimentos}

Ao apoio recebido do Programa de Pósgraduaçao do Banco Central do Brasil/ UniBacen e do Departamento de Medicina Social da Faculdade de Medicina da UFRGS.

Realizado no âmbito do Convênio CGDANT/SVS/Ministério da Saúde 3664/ 2005.

\section{Referências}

1. Murray CJL, Lopez AD. Global comparative assessments in the health sector: disease burden, expenditures and intervention packages. Geneva: World Health Organization, 1994.

2. Wild S, Roglic G, Green A, Sicree R, King H. Global prevalence of Diabetes: estimates for the year 2000 and projections for 2030. Diabetes Care 2004; 27(5):1047-53.

3. Barceló A, Rajpathak S. Incidence and prevalence of diabetes mellitus in the Americas. Pan American Journal of Public Health 2001; 10(5):300-8.

4. Malerbi DA, Franco LJ, The Brazilian Cooperative Group On The Study Of Diabetes Prevalence. Multicenter study of the prevalence of Diabetes Mellitus and Impaired Glucose Tolerance in the urban Brazilian population aged 30-69 yr. Diabetes Care 1992; 15(11):1509-16.
5. Nucci LB. A Campanha Nacional de Detecção do Diabetes Mellitus: cobertura e resultados glicêmicos. (tese de doutorado). Porto Alegre: Faculdade de Medicina da Universidade Federal do Rio Grande do Sul; 2003.

6. Monteiro CA, Mondini L, Souza ALM, Popkin BM. Da desnutrição para a obesidade: a transição nutricional no Brasil. In: Monteiro CA. (org.). Velhos e novos males da saúde no Brasil: a evolução do país e de suas doenças. São Paulo: Hucitec, Nupens/USP; 1995.

7. Gagliardino JJ, Williams R, Clark CM. Using hospitalization rates to track the economic costs and benefits of improved diabetes care in the Americas. Diabetes Care 2000; 23(12): 1844-6. 
8. Brasil/MS/DATASUS (Brasil. Ministério da Saúde. Datasus). Transferência de arquivos. Arquivos de Dados. Seleção de Arquivos do BBS. Disponível em: http:/ / datasus.gov.br. (Acessado em 20 de setembro de 2004)

9. Lessa FJD, Mendes ACG, Farias SF, Sá DA, Duarte PO, Melo Filho DA. Novas metodologias para vigilância epidemiológica: uso do Sistema de Informações Hospitalares - SIH/SUS. Informe Epidemiológico do SUS 2000; 9(S1): 3-27.

10. Brasil/IBGE (Brasil. Instituto Brasileiro de Geografia e Estatística). Censo Demográfico de 2000 - Resultados do Universo. Disponível em: http://www.ibge.gov.br. (Acessado em 6 de setembro de 2004)

11. Dever GEA. A epidemiologia na administração dos serviços de saúde. São Paulo: Pioneira, 1988.

12. King H, Aubert RE, Herman WH. Global burden of diabetes, 1995-2025 - prevalence, numerical estimates, and projections. Diabetes Care 1998; 21(9): 1414-31.

13. Jönsson B. Revealing the cost of Type II diabetes in Europe. Diabetologia 2002; (45): S5-S12.

14. ADA (American Diabetes Association). Economic costs of Diabetes in the U.S. in 2002. Diabetes Care 2003; 26(3): 917-32.

15. Barceló A, Aedo C, Rajpathak S, Robles S. The cost of diabetes in Latin America and the Caribbean. Bulletin of the World Health Organization 2003; 81(1): 19-27.

16. Hall MJ, Owings MF. 2000 National Hospital Discharge Survey. Advance Data from vital and health statistics 2002; 329 (jun. 19).

17. US Census Bureau (United States Census Bureau). United States Census 2000. Disponível em http:// www.census.gov (Acessado em 02 de dezembro de 2002)

18. OPAS (Organização Panamericana Da Saúde). RIPSA. Fontes de Informação: indicadores básicos para a saúde no Brasil - conceitos e aplicações. Brasília: OPAS, 2002. Apud: Malta DC, Cezário AC, Moura L, Morais Neto Ol, Silva Jr. JB. A construção da vigilância e prevenção das doenças crônicas não transmissíveis no contexto do Sistema Único de Saúde. Epidemiologia e Serviços de Saúde (ano de publicação) 15(3): 47-65.

19. Gadelha AMJ, Leite LC, Valente JG, Schramm JMA, Portela MC, Campos MR. Relatório Final do Projeto Estimativa da Carga de Doença no Brasil - 1998. Rio de Janeiro: FENSPTEC - Tecnologias em Saúde para a Qualidade de Vida, 2002.

20. Lima-Costa MFFL, Guerra Hl, Barreto SM, Guimarães RM. Diagnóstico da situação de saúde da população idosa brasileira: um estudo da mortalidade e das internações hospitalares públicas. Informe Epidemiológico do SUS 2000; 9(1): 23-41.

21. Bo S, Ciccone G, Grassi G, Gancia R, Rosato R, Merletti F, Pagano GF. Patients with type 2 diabetes had higher rates of hospitalization than the general population. Journal of Clinical Epidemiology 2004; 57: 1196-1201.
22. Travassos C, Viacava F, Pinheiro R, Brito A. Utilização dos serviços de saúde no Brasil: gênero, características familiares e condição social. Pan American Journal of Public Health 2002; 11(5/6): 365-73

23. Niefeld MR, Braunstein JB, Wu AW, Saudek CD, Weller WE, Anderson GF. Preventable hospitalization among eldery Medicare beneficiaries with type 2 diabetes. Diabetes Care 2003; 26(5): 1344-9.

24. Aro S, Koivisto VA, Reunanen A, Salinto M, Kangas T. Influence of morbidity and health care structure on hospitalization among adult diabetic patients. Diabetic Medicine 1995; 13(4): 376-81.

25. Krop JS, Saudek CD, Weller WE, Powe NR, Shaffer T, Anderson GF. Predicting expenditures for Medicare beneficiaries with diabetes - a prospective cohort study from 1994 to 1996. Diabetes Care 1999; 22(10): 1660-6.

26. Giuffrida A, Gravelle H, Roland M. Measuring quality of care with routine data: avoiding confusion between performance indicators and health outcomes. British Medical Journal 1999; 319(7202): 94-8.

27. Travassos C, Noronha Jc, Martins M. Mortalidade hospitalar como indicador de qualidade: uma revisão. Ciênc Saúde Coletiva 1999; 4(2):367-81.

28. Salles Gf, Bloch Kv, Cardoso Crl. Mortality and predictors of mortality in a cohort of Brazilian type 2 diabetic patients. Diabetes Care 2004; 27(6): 1299-305.

29. Franco Lj, Mameri C, Pagliaro H, Iochida Lc, Goldenberg P. Diabetes como causa básica ou associada de morte no Estado de São Paulo, Brasil, 1992. Rev Saúde Pública 1998; 32(3): 237-45.

30. Lima AK. Mortalidade por complicações agudas do Diabetes Mellitus no Brasil (dissertação de mestrado). Porto Alegre: Faculdade de Medicina, Universidade Federal do Rio Grande do Sul; 2006.

31. CDC (Centers For Disease Control And Prevention). Hospitalization for Diabetes as First-Listed Diagnosis. Number (in Thousands) of Hospital Discharges, Number (in Thousands) of Days of Hospital Stay, and Average Length of Stay (LOS) in Days for Discharges with Diabetes as First-Listed Diagnosis, by Age, United States, 1980-2002. Disponível em http://www.cdc.gov/diabetes/ statistics/ dmfirst/ byAgetable1_2.htm. (Acessado em 7 de janeiro de 2006)

32. Buss PM. Assistência hospitalar no Brasil (1984-1991): uma análise preliminar baseada no Sistema de Informação Hospitalar do SUS. Informe Epidemiológico do SUS 1993; ano II(2): 5-42.

33. ADA (American Diabetes Association). Economic consequences of Diabetes Mellitus in the U.S. in 1997. Diabetes Care 1998; 21(2): 296-309.

34. Andreazzi MFS. Formas de remuneração de serviços de saúde. IPEA: Brasília, 2003. [Texto para Discussão n 1006].

Recebido em: 29/01/07

Versão final reapresentada em: 10/08/07

Aprovado em: 24/08/07 